\section{La perspectiva de los agentes sanitarios sobre la incorporación programática de la autotoma del test de VPH}

\author{
Health agents' perspective on the incorporation \\ of self-collected samples in HPV screening \\ programs
}

\section{A perspectiva dos agentes sanitários sobre a incorporação programada da autoadministração do teste de HPV}

\section{Resumen}

El objetivo de este estudio fue analizar la percepción que poseen los agentes sanitarios sobre el ofrecimiento de la autotoma del test de VPH a las mujeres $y$ el grado de acuerdo de los agentes para incorporarla a sus tareas diarias. Para ello, se aplicó una encuesta auto-administrada a 127/191 agentes sanitarios que participaron del Proyecto EMA (Proyecto Evaluación Modalidad Autotoma), llevado a cabo en la provincia de Jujuy (Argentina) entre 2012-2013. Los agentes sanitarios que tuvieron y no la experiencia de ofrecer la autotoma manifestaron un alto grado de acuerdo para la adopción de la estrategia (78,7\%), dado su potencial para prevenir el cáncer cervicouterino y los aportes que brinda al cuidado de la salud de las mujeres bajo su cobertura. Sin embargo, señalaron la sobrecarga de trabajo y los problemas de articulación con el sistema formal de salud, como los principales obstáculos para ofrecer esta modalidad en el futuro. Este estudio encontró que la autotoma es una práctica que puede ser adoptada por los agentes sanitarios de la provincia de Jujuy, pero debe ir acompañada de acciones de apoyo por parte del sistema de salud formal.

Agentes Comunitarios de Salud; Papillomaviridae; Neoplasias del Cuello Uterino

\author{
Mariana Curotto 1 \\ Paula Barletta 1 \\ Melisa Paolino 1 \\ Silvina Arrossi 2,3
}

doi: 10.1590/0102-311X00138515

\author{
espondencia \\ S. Arrossi \\ Centro de Estudios de Estado y Sociedad. \\ Sánchez de Bustamante 27, Ciudad Autónoma de Buenos Aires \\ 1193, Argentina \\ silviarrossi2020@gmail.com \\ 1 Programa Nacional de Prevención de Cáncer Cervicouterino, \\ Instituto Nacional del Cáncer, Buenos Aires, Argentina. \\ 2 Centro de Estudios de Estado y Sociedad, Buenos Aires, \\ Argentina. \\ 3 Consejo Nacional de Investigaciones Científicas y Técnicas, \\ Buenos Aires, Argentina.
}




\section{Introducción}

A pesar de ser prevenible, el cáncer cervicouterino (CCU) es un problema significativo de salud pública, fundamentalmente en América Latina. En esta región es el segundo cáncer más diagnosticado en mujeres con una estimación de 69.000 nuevos casos y 28.600 muertes anuales ${ }^{1}$. En Argentina, cada año se diagnostican cerca de 5.000 nuevos casos y 1.800 mujeres mueren a causa de esta enfermedad 1 . La probabilidad de desarrollar este tipo de tumor se encuentra estrechamente relacionada con la participación de las mujeres en el tamizaje. En Argentina, el acceso al tamizaje de las mujeres socialmente más vulnerables se ha visto limitado por la existencia de barreras geográficas, culturales, sociales y vinculadas a la organización de los servicios de salud 2,3,4.

En los últimos años, el desarrollo de una nueva tecnología de tamizaje, basada en el ADN del virus del papiloma humano (VPH) cambió el escenario de la prevención, al brindar la posibilidad de la autotoma de la muestra por parte de las mujeres. La autotoma es altamente aceptable y posee un potencial para incrementar la participación en el tamizaje de aquellas mujeres que por vergüenza, temor a sentir dolor durante el examen físico, falta de tiempo, lejanía de los centros de salud y largos tiempos de espera para conseguir turnos no se tamizan de manera frecuente 5,6,7,8. Sin embargo, su incorporación a los programas de prevención requiere de la definición de aspectos vinculados a su implementación como - por ejemplo- quién realizará el ofrecimiento, dónde y cómo se entregarán los tubos colectores a las mujeres y, además, el modo en que se transportará al laboratorio para su procesamiento.

Si bien diversos estudios realizados en países de altos ingresos mostraron la eficacia del envío de la autotoma a las mujeres por carta 6,9,10 dicha estrategia no puede ser aplicada en Argentina por la ausencia de un listado nominalizado de las mujeres que no se tamizaron y por falencias del sistema postal 2 . En ese sentido, los agentes sanitarios se posicionan como un actor posible de realizar el ofrecimiento de la autotoma; ya que son uno de los pocos actores del sistema de salud que visita de manera rutinaria a las mujeres, de forma extra-hospitalaria y con una perspectiva netamente preventiva. Por otra parte, dado los criterios biomédicos con los cuales fueron formados y su activa participación en las prácticas de salud de la comunidad, los agentes sanitarios pueden cumplir un rol clave al explicar a las mujeres por qué y cómo deben realizarse la autotoma. De ese modo, los agentes sanitarios actúan como puente entre el saber técnico y los significados que la comunidad asigna a los procesos de saludenfermedad-atención, facilitando las prácticas de salud de la población bajo su cobertura 11,12,13,14.

En Argentina, el Proyecto EMA (Proyecto Evaluación Modalidad Autotoma) evaluó la efectividad del ofrecimiento de la autotoma a través de los agentes sanitarios jujeños, durante las visitas domiciliarias que realizan habitualmente. Los resultados del estudio mostraron que el tamizaje fue cuatro veces mayor en el grupo de mujeres a las cuales se les ofreció realizarse la autotoma, en comparación al grupo de mujeres entre las que se promocionó la realización de la prueba por un profesional en el centro de salud. Asimismo, el número de lesiones CIN2+ detectadas fue tres veces mayor entre las mujeres que se realizaron la autotoma que entre aquellas que se hicieron la toma convencional de VPH. El estudio concluyó que la autotoma ofrecida por los agentes sanitarios es una estrategia que puede ser incorporada por países de medianos y bajos ingresos para aumentar la cobertura del tamizaje, sobre todo entre aquellas mujeres con dificultades de acceso a los servicios de salud 15 . Este hallazgo también fue encontrado por dos estudios que evaluaron esta intervención con mujeres haitianas 16,17.

A pesar de que la estrategia mostró excelentes resultados, la sostenibilidad en el tiempo del ofrecimiento de la autotoma por parte de los agentes sanitarios, puede representar un desafío por el rol privilegiado que este actor ejerce en el contacto con la comunidad; lo que genera que el sistema de salud canalice en ellos la prestación de numerosos servicios y prácticas de cuidado de la salud, con el consiguiente riesgo de la sobrecarga de trabajo. Además, la sostenibilidad de esta estrategia puede verse afectada por factores como la desmotivación, la falta de incentivos y la percepción de los agentes sanitarios de una falta de apoyo por parte del sistema de salud; afectando los objetivos y metas planteados por los programas de tamizaje 18,19,20.

Teniendo en cuenta estos aspectos, este estudio se propuso analizar las percepciones y opiniones que poseen los agentes sanitarios que participaron del Proyecto EMA sobre el ofrecimiento de la autotoma y su grado de acuerdo para adoptarla como prestación de salud durante la ronda sanitaria. Conocer la perspectiva de los agentes sanitarios posibilitará identificar los obstáculos y facilitadores 
para realizar la labor, en el marco de la extensión territorial de la estrategia a toda la provincia de Jujuy y en otros contextos del país.

\section{Métodos}

\section{Ámbito de realización}

Jujuy está ubicada al noroeste de la Argentina y posee 673.307 habitantes, de los cuales el 51\% son mujeres y el $85 \%$ vive en áreas urbanas. El sistema de salud formal está constituido por un hospital central y 270 centros de atención primaria de la salud. En el 2011, en el marco del Proyecto Demostración para la Incorporación del Test de VPH como tamizaje primario en Argentina 21, Jujuy incorporó en el sistema público esta nueva modalidad para prevenir el CCU. La prueba es ofrecida de manera gratuita a todas las mujeres de 30 y más años.

En lo que respecta específicamente al sistema provincial de atención primaria de la salud, su origen se remonta a 1966 cuando se implementó el Plan de Salud Rural a cargo del Dr. Carlos Alvarado 22. En la actualidad, Jujuy cuenta con una dirección provincial de atención primaria de la salud y seis grandes regiones sanitarias que poseen un supervisor general a cargo. Para realizar la labor territorial, la provincia emplea alrededor de 700 agentes sanitarios que trabajan tiempo completo y reciben un salario por su labor. El total de los agentes sanitarios visita aproximadamente 110.000 hogares durante sus rondas sanitarias, en las cuales realizan tareas que incluyen la inmunización y promoción del cuidado materno-infantil. Su desempeño es evaluado de manera anual por los supervisores de atención primaria de la salud, de acuerdo con las metas establecidas.

\section{Población de estudio, recolección y análisis de los datos}

Esta investigación forma parte del Proyecto EMA, que se llevó a cabo entre 2012-2013 en la provincia de Jujuy 15. El Proyecto EMA consistió en un estudio de métodos combinados, compuesto por un estudio randomizado por clusters 15 , entrevistas cualitativas a mujeres y una encuesta transversal a los agentes sanitarios, cuyos resultados se reportan en el presente artículo. En el marco del Proyecto EMA, 200 agentes sanitarios fueron seleccionados y asignados 1:1 para ofrecer durante la visita domiciliaria la autotoma a la mujer (Grupo Autotoma-GAT) o promover la realización de la prueba por un profesional en el centro de la salud (Grupo Toma Convencional-GTC). Un total de 191 agentes sanitarios participaron del estudio ( 94 en el GAT y 97 en el GTC) y fueron capacitados sobre la prevención del CCU, el test de VPH y el protocolo de investigación. Los agentes sanitarios del GAT recibieron una capacitación adicional sobre estrategias de comunicación para poder explicar a la mujer cómo realizarse la autotoma. Al momento de la implementación de la autotoma, los agentes sanitarios de ambos grupos identificaron a las mujeres elegibles en sus hogares durante las rondas sanitarias que habitualmente realizan y, al contactarlas, las invitaron a participar del estudio. Los agentes sanitarios del GAT ofrecieron a las mujeres la posibilidad de realizarse la autotoma en la intimidad de su hogar y en el caso de que no aceptaran se promovía la realización del test de VPH en los centros de salud. Los agentes sanitarios del GTC solo promovieron la realización del test en los centros de salud. El Proyecto EMA fue aprobado para su realización por el Comité Provincial de Ética de la Investigación en Salud de Jujuy.

Para poder responder específicamente al objetivo de describir las percepciones y opiniones que poseen los agentes sanitarios sobre la adopción del ofrecimiento de la autotoma como tarea habitual, se llevó adelante una encuesta transversal que tuvo como población objetivo al total de agentes sanitarios que participaron del Proyecto EMA. Se aplicó un cuestionario anónimo y auto-administrado, específicamente construido para este estudio, en base a la experiencia de trabajo en la provincia y la revisión de literatura 11,12,13,14,15,16,17,18,19,20. Se trató de un cuestionario estructurado, compuesto por preguntas cerradas y abiertas que indagaron acerca de aspectos positivos/negativos de ofrecer la autotoma, grado de acuerdo/desacuerdo para incorporar la autotoma como tarea, razones para incorporar/no incorporar la autotoma, y obstáculos/facilitadores para ofrecer la autotoma en el 
futuro. Las respuestas a las preguntas abiertas fueron posteriormente recodificadas según la literatura existente sobre la autotoma 5,6,7,8,9,10 y sobre agentes sanitarios 11,12,13,14,15,16,17,18,19,20. Asimismo, el cuestionario incluyó las variables sexo, área de trabajo y Grupo Modalidad de Ofrecimiento (GMO) para caracterizar a la población entrevistada.

La encuesta fue implementada en diciembre del 2012 en la ciudad de San Salvador de Jujuy, en el marco del taller de cierre de la etapa de reclutamiento de mujeres para el Proyecto EMA. Dado que la incorporación programática de la autotoma se planeaba implementar con la totalidad de los agentes sanitarios de Jujuy que -en su mayoría- no habían participado del Proyecto EMA, el cuestionario fue administrado tanto a los agentes sanitarios del GAT (quienes tuvieron la experiencia de ofrecer la autotoma), como a los agentes sanitarios del GTC (quienes no tuvieron dicha experiencia, pero podían inferirla de lo contado por sus compañeros del GAT). La encuesta fue entregada a los agentes sanitarios al comienzo del taller; momento en el cual fueron explicados sus objetivos y el modo de completarla. Se pautó utilizar el espacio de intervalo del taller para su concreción y posterior entrega.

\section{Análisis}

Se realizaron frecuencias simples y porcentajes para cada una de las variables de estudio. Los porcentajes fueron elaborados sobre el total de respuestas obtenidas según GMO. Asimismo, se realizaron comparaciones entre los agentes sanitarios entrevistados y los no entrevistados y, también, entre el grupo que manifestó estar de acuerdo y el grupo en desacuerdo con la incorporación de la autotoma, según sexo, área de trabajo y GMO. La asociación entre las variables fue evaluada mediante el test de independencia chi cuadrado $\left(\chi^{2}\right)$. La significación estadística fue establecida en 0,05.

\section{Resultados}

De los 191 agentes sanitarios que fueron convocados al taller de cierre de las capacitaciones del Proyecto EMA, 127 completaron la encuesta auto-administrada y conforman la muestra de este estudio. Las características demográficas de los 127 agentes sanitarios se presentan en la Tabla 1: 81,1\% eran mujeres; 74,8\% realizaban sus labores en áreas urbanas; 57,5\% pertenecían al GAT y 42,5\% al GTC. No se encontraron diferencias estadísticamente significativas entre los grupos, con relación al sexo y área, pero sí con respecto al GMO.

\section{Aspectos positivos y negativos de ofrecer la autotoma}

El aspecto positivo de la autotoma más mencionado por los agentes sanitarios fue que "permite ampliar la cobertura de tamizaje, mejorando la prevención del CCU”; mención que concentró el 32,2\% de las respuestas de los agentes y que, además, obtuvo similar frecuencia de respuesta en el GAT (31,6\%) y en el GTC (33,1\%). En segundo y tercer lugar, los agentes sanitarios señalaron que la autotoma "ahorra tiempo y traslado favoreciendo la accesibilidad al sistema de salud" $(16,8 \%)$ y que "aporta a la privacidad y practicidad de las mujeres" (16\%). Dichas categorías fueron menos mencionadas en el GAT (14\% y $12,7 \%)$, en comparación al GTC (20,8\% para ambas categorías). Por otro lado, se destaca que la identificación de la autotoma como una "práctica aceptada por las mujeres" $(8,9 \%)$ y que "fortalece el vínculo agente sanitario-mujer" (7,3\%) fue mayor en el GAT (12,7\% y 9,6\%) que en el GTC (3,2\% y 3,9\%) (Tabla 2).

En lo que respecta a los aspectos negativos, el más mencionado por los agentes sanitarios fue la "sobrecarga de trabajo". Esta mención concentró el 32\% de las respuestas de los agentes sanitarios y obtuvo similar frecuencia de respuesta en el GAT $(31,8 \%)$ y en el GTC (32,3\%). En segundo lugar, el $24,9 \%$ de los agentes sanitarios indicaron "rechazo o temor de la mujer para realizar la autotoma". Esta respuesta fue más mencionada en el GAT (27,3\%), en comparación al GTC (21,2\%). En tercer lugar, los agentes sanitarios señalaron "problemas de articulación y/o vínculo con el sistema de salud" (17,8\%); siendo mayor esta mención en el GAT (20,8\%) que en el GTC (13,1\%). Por otro lado, las categorías "dificultades para explicar la autotoma por falta de capacitación" (5,9\%) y "no contar con el kit de la autotoma durante la visita” (4\%) fueron respuestas menos señaladas en el GAT (1,9\% y 2,6\%) que en el GTC (12,1\% y 6,1\%) (Tabla 2). 
Tabla 1

Características demográficas de los agentes sanitarios que participaron de la encuesta.

\begin{tabular}{|c|c|c|c|c|c|c|c|}
\hline & \multicolumn{2}{|c|}{$\begin{array}{l}\text { Agentes sanitarios } \\
\text { Proyecto EMA }\end{array}$} & \multicolumn{2}{|c|}{$\begin{array}{l}\text { Agentes sanitarios } \\
\text { presentes en la encuesta }\end{array}$} & \multicolumn{2}{|c|}{$\begin{array}{l}\text { Agentes sanitarios } \\
\text { ausentes en la encuesta }\end{array}$} & \multirow[t]{2}{*}{ Valor de $p$} \\
\hline & $\mathbf{n}$ & $\%$ & $\mathbf{n}$ & $\%$ & $\mathbf{n}$ & $\%$ & \\
\hline Total & 191 & 100,0 & 127 & 100,0 & 64 & 100,0 & \\
\hline Sexo & & & & & & & 0,092 \\
\hline Masculino & 43 & 22,5 & 24 & 18,9 & 19 & 29,7 & \\
\hline Femenino & 148 & 77,5 & 103 & 81,1 & 45 & 70,3 & \\
\hline Área & & & & & & & 0,091 \\
\hline Urbano & 134 & 70,2 & 95 & 74,8 & 39 & 60,9 & \\
\hline Rural & 56 & 29,3 & 31 & 24,4 & 25 & 39,1 & \\
\hline Sin identificar & 1 * & 0,5 & 1 & 0,8 & & & \\
\hline Grupo Modalidad de Ofrecimiento (GMO) & & & & & & & 0,001 \\
\hline Autotoma & 94 & 49,2 & 73 & 57,5 & 21 & 32,8 & \\
\hline Toma convencional & 97 & 50,8 & 54 & 42,5 & 43 & 67,2 & \\
\hline
\end{tabular}

* Se perdió el dato de un agente sanitario.

Tabla 2

Percepción de aspectos positivos y negativos de ofrecer la autotoma, según Grupo Modalidad de Ofrecimiento (GMO) *.

\begin{tabular}{|c|c|c|c|c|c|c|}
\hline \multirow{2}{*}{ Respuestas de los agentes sanitarios } & \multicolumn{2}{|c|}{ Total } & \multicolumn{2}{|c|}{ GAT } & \multicolumn{2}{|c|}{ GTC } \\
\hline & $\mathrm{n}$ & $\%$ & $\mathbf{n}$ & $\%$ & $\mathbf{n}$ & $\%$ \\
\hline \multicolumn{7}{|l|}{ Aspectos positivos de ofrecer la autotoma } \\
\hline $\begin{array}{l}\text { Permite ampliar la cobertura de tamizaje, mejorando la prevención del cáncer } \\
\text { cervicouterino }\end{array}$ & 123 & 32,2 & 72 & 31,6 & 51 & 33,1 \\
\hline Ahorra tiempo y traslado, favoreciendo la accesibilidad al sistema de salud & 64 & 16,8 & 32 & 14,0 & 32 & 20,8 \\
\hline Aporta a la privacidad y practicidad de las mujeres & 61 & 16,0 & 29 & 12,7 & 32 & 20,8 \\
\hline Brinda mejor calidad de atención a la comunidad & 43 & 11,3 & 27 & 11,8 & 16 & 10,4 \\
\hline Es una práctica aceptada por las mujeres & 34 & 8,9 & 29 & 12,7 & 5 & 3,2 \\
\hline Fortalece el vínculo agente sanitario-mujer & 28 & 7,3 & 22 & 9,6 & 6 & 3,9 \\
\hline Brinda satisfacción personal al agente sanitario & 5 & 1,3 & 5 & 2,2 & 0 & 0,0 \\
\hline Otras respuestas & 19 & 5,0 & 11 & 4,8 & 8 & 5,2 \\
\hline No sabe/No contesta & 5 & 1,3 & 1 & 0,4 & 4 & 2,6 \\
\hline Total de respuestas & 382 & 100,0 & 228 & 100,0 & 154 & 100,0 \\
\hline \multicolumn{7}{|l|}{ Aspectos negativos de ofrecer la autotoma } \\
\hline Sobrecarga de trabajo & 81 & 32,0 & 49 & 31,8 & 32 & 32,3 \\
\hline Rechazo o temor de la mujer para realizar la autotoma & 63 & 24,9 & 42 & 27,3 & 21 & 21,2 \\
\hline Problemas de articulación/vínculo con el sistema de salud & 45 & 17,8 & 32 & 20,8 & 13 & 13,1 \\
\hline Dificultades para explicar la autotoma por falta de capacitación & 15 & 5,9 & 3 & 1,9 & 12 & 12,1 \\
\hline No contar con el kit de la autotoma durante la visita & 10 & 4,0 & 4 & 2,6 & 6 & 6,1 \\
\hline Sin aspectos negativos & 10 & 4,0 & 8 & 5,2 & 2 & 2,0 \\
\hline Otras respuestas & 9 & 3,6 & 9 & 5,8 & 0 & 0,0 \\
\hline No sabe/No contesta & 20 & 7,9 & 7 & 4,5 & 13 & 13,1 \\
\hline Total de respuestas & 253 & 100,0 & 154 & 100,0 & 99 & 100,0 \\
\hline
\end{tabular}

GAT: Grupo Autotoma; GTC: Grupo Toma Convencional.

* Respuesta espontánea múltiple/porcentajes basados en casos. 


\section{Grado de acuerdo y/o desacuerdo para incorporar la autotoma a las tareas diarias}

El 78,7\% del total de los agentes sanitarios manifestó estar de acuerdo con la incorporación de la autotoma a sus tareas diarias. El grado de acuerdo fue mayor en el GAT (87,8\%) que en el GTC (66\%); siendo esta diferencia estadísticamente significativa $(\mathrm{p}=0,004)$ (Tabla 3$)$. Si se analiza el perfil de los agentes sanitarios que estuvieron de acuerdo en incorporar la autotoma, se observa que la mayoría eran mujeres (80\%); trabajaban en zonas urbanas (72\%) y habían participado del GAT (65\%). No se encontraron diferencias estadísticamente significativas entre los grupos según sexo y área, pero sí según GMO; observándose una mayor proporción de agentes sanitarios del GAT entre el grupo que manifestó acuerdo con la adopción de la estrategia (65\%; p = 0,003) (Tabla 4).

$\mathrm{Al}$ momento de brindar las razones de acuerdo para incorporar la autotoma, los agentes sanitarios indicaron en primer lugar que "permite ampliar la cobertura del tamizaje, mejorando la prevención del CCU" (31,5\%). Esta mención concentró el 32,7\% de las respuestas del GAT y el 29,1\% del GTC. En segundo lugar, el 21,4\% de los agentes sabitarios indicaron que la autotoma "aporta a la privacidad y practicidad de las mujeres”; respuesta que fue mayor en el GAT (24,8\%) que en el GTC (14,5\%). En tercer lugar, los agentes sabnitarios mencionaron que la autotoma "ahorra tiempo y traslado favoreciendo la accesibilidad al sistema de salud” (20,2\%); mención que fue indicada por el 22,1\% del GAT y el 16,4\% del GTC. En lo que respecta a las razones para estar en desacuerdo de incorporar la autotoma, el 44,9\% de los agentes sanitarios señaló a la "sobrecarga de trabajo"; sin grandes diferencias entre el GAT $(47,6 \%)$ y el GTC (42,9\%). En segundo lugar, los agentes sanitarios indicaron como motivo el "rechazo o temor de la mujer para realizar la autotoma” (14,3\%) y, en tercer lugar, "hay mujeres que prefieren ser atendidas por un profesional" (10,2\%). Si se analiza según GMO para ambas categorías, se observa que el total de las respuestas pertenecen al GTC (25\% y $17,9 \%)$ (Tabla 3$)$.

\section{Facilitadores y obstaculizadores de la tarea de ofrecer la autotoma}

El facilitador más mencionado por los agentes sanitarios fue "mejorar la articulación y/o vínculo con el sistema de salud" (20,7\%); el cual fue indicado por el 25,2\% del GAT y el 14,6\% del GTC. En segundo lugar, los agentes sanitarios indicaron "planificar la búsqueda y seguimiento de mujeres sin tamizaje o con resultado $\mathrm{VPH}+$ ” (15\%); categoría que obtuvo una menor frecuencia de respuestas en el GAT $(13,5 \%)$ que en el GTC (17,1\%). En tercer lugar, los agentes señalaron que "redistribuir los tiempos y las tareas de los agentes sanitarios" (14\%) podría facilitar la tarea de ofrecer la autotoma en el futuro; este facilitador concentró el 17,1\% de las respuestas del GAT y el 9,8\% del GTC. Por otro lado, se resalta que "contar con el kit durante la visita domiciliaria" (9,8\%) fue un facilitador poco mencionado en el GAT (6,3\%) en comparación al GTC (14,6\%). Mientras que "generar buenas formas de comunicación con la mujer para fortalecer la confianza en el agente sanitario" (6,2\%) fue una demanda mayor en el GAT (9\%) que en el GTC (2,4\%) (Tabla 5).

En lo que respecta a los obstáculos de ofrecer la autotoma, el 27,9\% de los agentes sanitarios contestó "ninguno"; respuesta que fue brindada en mayor medida por el GAT $(35,6 \%)$ que por el GTC (18,7\%). En segundo lugar, los agentes sanitarios señalaron a la "sobrecarga de trabajo" (26,1\%); concentrando el 28,9\% de las respuestas del GAT y el 22,7\% del GTC. En tercer lugar, los agentes sanitarios indicaron la existencia de "problemas de articulación y/o vínculo con el sistema de salud" (12,7\%); mención que obtuvo similar frecuencia de respuestas en el GAT (12,2\%) y en el GTC (13,3\%). Por otro lado, se destaca que "no contar con el kit durante la visita" (9,1\%), "falta de capacitación por parte de los agentes sanitarios que no la han ofrecido" (6,7\%) y "rechazo o temor de la mujer para realizar la autotoma" (5,5\%) fueron respuestas menos frecuentes en el GAT (6,7\%; 4,4\%; 1,1\%) que en el GTC (12\%; 9,3\%; 10,7\%) (Tabla 5).

\section{Discusión}

Si bien diversos estudios han indagado sobre diferentes aspectos de la autotoma 6,10,15,23,24, este trabajo es el primero en relevar la voz de los agentes sanitarios; actores clave para su implementación programática en los países de medianos y bajos ingresos. En ese sentido, esta investigación constituye un aporte para la identificación de los desafíos con los que la estrategia del ofrecimiento de la autotoma a 
Tabla 3

Grado de acuerdo y/o desacuerdo para incorporar la autotoma a las tareas diarias (y razones de ello), según Grupo Modalidad Ofrecimiento (GMO).

\begin{tabular}{|c|c|c|c|c|c|c|}
\hline \multirow[t]{2}{*}{ Respuestas de los agentes sanitarios } & \multicolumn{2}{|c|}{ Total } & \multicolumn{2}{|c|}{ GAT * } & \multicolumn{2}{|c|}{ GTC * } \\
\hline & $\mathbf{n}$ & $\%$ & $\mathbf{n}$ & $\%$ & $\mathbf{n}$ & $\%$ \\
\hline \multicolumn{7}{|l|}{ Grado de acuerdo y desacuerdo para incorporar la autotoma a las tareas diarias ** } \\
\hline De acuerdo & 100 & 78,7 & 65 & 87,8 & 35 & 66,0 \\
\hline Ni de acuerdo ni en desacuerdo & 15 & 11,8 & 3 & 4,1 & 12 & 22,6 \\
\hline En desacuerdo & 12 & 9,4 & 6 & 8,1 & 6 & 11,3 \\
\hline Total de respuestas & 127 & 100,0 & 74 & 100 & 53 & 100,0 \\
\hline \multicolumn{7}{|l|}{ Razones de acuerdo para incorporar la autotoma ** } \\
\hline $\begin{array}{l}\text { Permite ampliar la cobertura de tamizaje, mejorando la prevención del cáncer } \\
\text { cervicouterino }\end{array}$ & 53 & 31,5 & 37 & 32,7 & 16 & 29,1 \\
\hline Aporta a la privacidad y practicidad de las mujeres & 36 & 21,4 & 28 & 24,8 & 8 & 14,5 \\
\hline Ahorra tiempo y traslado, favoreciendo la accesibilidad al sistema de salud & 34 & 20,2 & 25 & 22,1 & 9 & 16,4 \\
\hline Brinda mejor calidad de atención a la comunidad & 19 & 11,3 & 10 & 8,8 & 9 & 16,4 \\
\hline Es una práctica aceptada por las mujeres & 11 & 6,5 & 6 & 5,3 & 5 & 9,1 \\
\hline Fortalece el vínculo agente sanitario/mujer & 5 & 3,0 & 2 & 1,8 & 3 & 5,5 \\
\hline Brinda satisfacción personal al agente sanitario & 3 & 1,8 & 0 & 0,0 & 3 & 5,5 \\
\hline Otras respuestas & 7 & 4,2 & 5 & 4,4 & 2 & 3,6 \\
\hline Total de respuestas & 168 & 100,0 & 113 & 100,0 & 55 & 100,0 \\
\hline \multicolumn{7}{|l|}{ Razones en desacuerdo para incorporar la autotoma a las tareas diarias $* * *$} \\
\hline Sobrecarga de trabajo & 22 & 44,9 & 10 & 47,6 & 12 & 42,9 \\
\hline Rechazo o temor de la mujer para realizar la autotoma & 7 & 14,3 & 0 & 0,0 & 7 & 25,0 \\
\hline Hay mujeres que prefieren ser atendidas por un profesional & 5 & 10,2 & 0 & 0,0 & 5 & 17,9 \\
\hline $\begin{array}{l}\text { Falta de recursos humanos en el sistema de salud para colaborar en la tarea de ofrecer la } \\
\text { autotoma }\end{array}$ & 4 & 8,2 & 4 & 19,0 & 0 & 0,0 \\
\hline No sabe/No contesta & 11 & 22,4 & 7 & 33,3 & 4 & 14,3 \\
\hline Total de respuestas & 49 & 100,0 & 21 & 100,0 & 28 & 100,0 \\
\hline
\end{tabular}

GAT: Grupo Autotoma; GTC: Grupo Toma Convencional.

* Valor de $\mathrm{p}=0,004$;

** Respuesta guiada simple;

$\star \star \star *$ Respuesta espontánea múltiple/porcentaje basados en casos.

\section{Tabla 4}

Características de los agentes sanitarios que están de acuerdo en incorporar la autotoma a sus tareas diarias.

\begin{tabular}{|c|c|c|c|c|c|}
\hline & \multicolumn{2}{|c|}{$\begin{array}{c}\text { Agentes sanitarios presentes en la } \\
\text { encuesta }\end{array}$} & \multicolumn{2}{|c|}{ Agentes sanitarios "de acuerdo" } & \multirow[t]{2}{*}{ Valor de $p$} \\
\hline & $\mathbf{n}$ & $\%$ & $\mathbf{n}$ & $\%$ & \\
\hline Total & 127 & 100,0 & 100 & 100,0 & \\
\hline Sexo & & & & & 0,864 \\
\hline Masculino & 25 & 19,7 & 20 & 20,0 & \\
\hline Femenino & 102 & 80,3 & 80 & 80,0 & \\
\hline Área & & & & & 0,567 \\
\hline Urbano & 94 & 74,0 & 72 & 72,0 & \\
\hline Rural & 32 & 25,2 & 27 & 27,0 & \\
\hline Grupo Modalidad de Ofrecimiento (GMO) & & & & & 0,003 \\
\hline Autotoma & 74 & 58,3 & 65 & 65,0 & \\
\hline Toma convencional & 53 & 41,7 & 35 & 35,0 & \\
\hline
\end{tabular}




\section{Tabla 5}

Facilitadores y obstaculizadores de la tarea de ofrecer la autotoma, según Grupo Modalidad de Ofrecimiento (GMO) *.

\begin{tabular}{|c|c|c|c|c|c|c|}
\hline \multirow[t]{2}{*}{ Respuestas de los agentes sanitarios } & \multicolumn{2}{|c|}{ Total } & \multicolumn{2}{|c|}{ GAT } & \multicolumn{2}{|c|}{ GTC } \\
\hline & $\mathbf{n}$ & $\%$ & $\mathbf{n}$ & $\%$ & $\mathbf{n}$ & $\%$ \\
\hline \multicolumn{7}{|l|}{ Facilitadores de la tarea de ofrecer la autotoma } \\
\hline Mejorar la articulación/vínculo con el sistema de salud & 40 & 20,7 & 28 & 25,2 & 12 & 14,6 \\
\hline Planificar la búsqueda y seguimiento de mujeres sin tamizaje o con resultado VPH positivo & 29 & 15,0 & 15 & 13,5 & 14 & 17,1 \\
\hline Redistribuir los tiempos y tareas de los agentes sanitarios & 27 & 14,0 & 19 & 17,1 & 8 & 9,8 \\
\hline Contar con el kit durante la visita domiciliaria & 19 & 9,8 & 7 & 6,3 & 12 & 14,6 \\
\hline Impulsar campañas de comunicación que promuevan la autotoma & 17 & 8,8 & 7 & 6,3 & 10 & 12,2 \\
\hline $\begin{array}{l}\text { Generar buenas formas de comunicación con la mujer para fortalecer la confianza en el } \\
\text { agente sanitario }\end{array}$ & 12 & 6,2 & 10 & 9,0 & 2 & 2,4 \\
\hline Brindar incentivos (vehículos y remuneración económica) & 9 & 4,7 & 7 & 6,3 & 2 & 2,4 \\
\hline Implementar la autotoma a nivel provincial & 4 & 2,1 & 4 & 3,6 & 0 & 0,0 \\
\hline Otras respuestas & 20 & 10,4 & 5 & 4,5 & 15 & 18,3 \\
\hline No sabe/No contesta & 16 & 8,3 & 9 & 8,1 & 7 & 8,5 \\
\hline Total de respuestas & 193 & 100,0 & 111 & 100,0 & 82 & 100,0 \\
\hline \multicolumn{7}{|l|}{ Obstaculizadores de la tarea de ofrecer la autotoma } \\
\hline Ninguno & 46 & 27,9 & 32 & 35,6 & 14 & 18,7 \\
\hline Sobrecarga de trabajo & 43 & 26,1 & 26 & 28,9 & 17 & 22,7 \\
\hline Problemas de articulación/vínculo con el sistema de salud & 21 & 12,7 & 11 & 12,2 & 10 & 13,3 \\
\hline No contar con el kit durante la visita & 15 & 9,1 & 6 & 6,7 & 9 & 12,0 \\
\hline Falta de capacitación por parte de los agentes sanitarios que no la han ofrecido & 11 & 6,7 & 4 & 4,4 & 7 & 9,3 \\
\hline Rechazo o temor de la mujer para realizar la autotoma & 9 & 5,5 & 1 & 1,1 & 8 & 10,7 \\
\hline No contar con vehículo para poder llegar a domicilios alejados y trasladar las muestras & 8 & 4,8 & 5 & 5,6 & 3 & 4,0 \\
\hline No sabe/No contesta & 12 & 7,3 & 5 & 5,6 & 7 & 9,3 \\
\hline Total de respuestas & 165 & 100,0 & 90 & 100,0 & 75 & 100,0 \\
\hline
\end{tabular}

GAT: Grupo Autotoma; GTC: Grupo Toma Convencional.

* Respuesta espontánea múltiple/porcentajes basados en casos.

las mujeres a través de los agentes sanitarios, puede enfrentarse cuando es puesta en funcionamiento en condiciones reales de aplicación.

En lo que respecta al grado de acuerdo para la adopción de la estrategia por parte de los agentes sanitarios, nuestro estudio mostró que -pese al reconocimiento de la existencia de diferentes obstáculos para realizar la labor- la mayoría de los agentes sanitarios estaría de acuerdo en ofrecer la autotoma como tarea programática adicional a las que habitualmente realizan. Los motivos más mencionados por los agentes sanitarios se relacionaron con los aportes que esta nueva modalidad de tamizaje brinda al cuidado de la salud de las mujeres: privacidad, practicidad, ahorro de tiempo y traslado. La literatura sobre los agentes sanitarios reconoce que ofrecer mejoras en la calidad de vida, de los miembros de las comunidades en la cual viven y trabajan, es un factor de motivación para la elección de su rol profesional y la incorporación de nuevas tareas 14,19,25,26. Este estudio también encontró que la autotoma es considerada por los agentes sanitarios como una tecnología con el potencial de mejorar el acceso y cuidado de la salud de las mujeres; razón que funcionó como motivador para adoptar esta tarea como una más entre las que actualmente realizan.

Por otro lado, diversas investigaciones han visibilizado que la construcción de un vínculo de confianza entre los agentes sanitarios y la comunidad es fundamental para la efectividad de la labor de los agentes, la aceptabilidad de los programas por parte de los destinatarios y, además, para generar procesos de empoderamiento en la comunidad para la realización de sus prácticas de salud 13,14,19,20,27. En nuestro estudio, el fortalecimiento del vínculo fue considerado especialmente por los agentes sanitarios que participaron del GAT como un aspecto positivo de haber ofrecido la autotoma. El reco- 
nocimiento de la comunidad sobre la labor de los agentes sanitarios funciona como incentivo para la realización de las tareas de los agentes 14,27 y, en algunos casos, dicho reconocimiento suplementa la ausencia de incentivos por parte del sistema formal de salud 28 . En el contexto jujeño, la mención de los agentes sanitarios sobre el fortalecimiento de su vínculo con las mujeres a su cargo fue superior a la demanda de incentivos materiales (monetarios/no monetarios). En ese sentido, este estudio disiente con otros en los que la ausencia de incentivos materiales fueron el principal obstáculo percibido por los agentes sanitarios para la implementación y/o sostenibilidad de una estrategia 14,28,29.

Sin embargo, el vínculo entre los agentes sanitarios y las mujeres no es algo que se construya de una vez para siempre. Es un proceso de construcción permanente que necesita alcanzar un equilibrio entre las expectativas, el respeto, la credibilidad y la legitimidad entre ambos. Para ello, son necesarias ciertas habilidades por parte de los agentes sanitarios; así como un entrenamiento constante que permita desarrollarlas en forma procesual, integrando el conocimiento técnico-médico con el de la comunidad 14,19,27. En lo que respecta específicamente a la autotoma, desarrollar buenas pautas de comunicación es clave para entablar el vínculo con la mujer; ya que la prevención del CCU involucra temáticas ligadas a la sexualidad y al cáncer que suelen generar sentimientos de incomodidad, vergüenza y temores en la mujer 30 . Además, la especificidad del ofrecimiento no sólo supone poder explicar qué es la enfermedad y cómo puede prevenirse, sino cómo debe realizarse el test y entenderse su resultado. Este estudio encontró que los agentes sanitarios del GAT, quienes recibieron una capacitación especial para explicar a la mujer cómo realizar la autotoma, indicaron con menor frecuencia la existencia de problemas en el ofrecimiento por falta de capacitación. No obstante, los agentes sanitarios encontraron dificultades ante el rechazo y/o temor de la mujer de realizarse por sí misma la prueba. La negativa de la mujer, señalada como aspecto negativo de haber ofrecido la autotoma, indica una frustración por parte de los agentes sanitarios. Un estudio pakistaní relevó que -ante las barreras percibidas por la comunidad para la implementación de estrategias preventivas- los agentes sanitarios demandaron mayor conocimiento y entrenamiento en habilidades comunicacionales para poder enfrentarlas y superarlas 31 . Esta demanda también estuvo presente en los agentes jujeños, especialmente en el GAT, cuando se indagó sobre cuáles serían los facilitadores de ofrecer la autotoma. En ese sentido, se señala la importancia de profundizar la capacitación de los agentes sanitarios sobre las barreras subjetivas que pueden tener las mujeres para tamizarse, y también su entrenamiento en técnicas comunicacionales, para evitar que la negativa de la mujer actúe como un desmotivador de la labor de los agentes.

La construcción de un vínculo entre los agentes sanitarios y la comunidad es fundamental para el buen desempeño de los programas basados en los agentes, pero por sí sólo no alcanza. Es también esencial poder entablar un vínculo sostenido en el tiempo entre los agentes y el sistema formal de salud. Al respecto, la literatura destaca el esfuerzo que debe realizarse en pos de una adecuada planificación e integración de las tareas de los agentes con las del equipo de salud y con los niveles de gobierno para legitimar su trabajo y contar con recursos para desarrollar su labor 14,32. Para lograr dicha articulación los programas deben poder delimitar los roles y tareas asignados a los agentes sanitarios; definir metas posibles de cumplir; brindar capacitación sostenida en el tiempo; acompañar su trabajo territorial mediante la supervisión y el estímulo, además de garantizar los insumos para poder ofrecer una buena atención a la comunidad 14,19,26,33,34. La ausencia de alguno o varios de estos aspectos resultarán en una posible disminución de la confianza construida entre los agentes sanitarios y la comunidad y, asimismo, podrán generar frustración en los agentes, al no poder contribuir a elevar la calidad de vida de la población bajo su cobertura 19,34.

En el momento de ser consultados sobre los obstáculos y facilitadores de la tarea de ofrecer la autotoma, los agentes sanitarios señalaron la necesidad de mejorar la articulación con el sistema de salud antes y después de realizado el ofrecimiento. En lo que respecta a la organización de la estrategia, los agentes sanitarios de ambos grupos comparten la idea de que la planificación de la búsqueda activa y el seguimiento de las mujeres sin tamizaje y/o con resultados $\mathrm{VPH}+$ podrían facilitar esta tarea. Los agentes también mencionaron la importancia del buen funcionamiento del circuito de la entrega de tubos, al indicar que contar con el kit durante la visita facilitaría el ofrecimiento. La literatura sobre los agentes sanitarios destaca que la limitación en el acceso a insumos por los mismos puede afectar el servicio de salud puerta-a-puerta 14 , trayendo como consecuencia el sentimiento de vergüenza en los trabajadores y la pérdida de la credibilidad de los agentes ante la comunidad 34,35. En el caso del 
Proyecto EMA, el monitoreo realizado por el equipo de investigación no encontró problemas en la entrega de los tubos a los agentes sanitarios. Ello coincide con el hecho de que la posible carencia de insumos fue mencionada como un potencial obstaculizador -sobre todo- por los agentes del GTC; es decir, por aquellos que no tuvieron la experiencia de haber ofrecido la autotoma. De todos modos, esto subraya la importancia de planificar y monitorear la accesibilidad de los agentes sanitarios al kit de la autotoma, porque los problemas logísticos y/o burocráticos pueden poner en riesgo la estrategia y, además, la credibilidad de los agentes ante las mujeres bajo su cobertura.

Por otro lado, los agentes también hicieron mención a problemas de articulación con el sistema de salud en instancias posteriores al ofrecimiento de la autotoma, como demoras en la entrega de resultados, la organización de turnos de las derivaciones de las mujeres VPH+ y la falta de cooperación por parte de algunos profesionales del equipo de salud. Estos aspectos fueron especialmente indicados por los agentes del GAT, quienes también demandaron la redistribución de los tiempos y tareas. Este hallazgo coincide con un estudio realizado en Irán 36 que evidenció que la ausencia de apoyo, por parte del sistema de salud a los agentes sanitarios, y la sobrecarga de trabajo fueron las principales barreras identificadas por los agentes para la realización de su labor. En lo que respecta específicamente a la sobrecarga de trabajo, los agentes participantes de este estudio -sin importar el GMO- la señalaron como el principal aspecto negativo de haber ofrecido la autotoma, la principal razón para no incorporarla y el principal obstaculizador de la tarea. La sobrecarga de trabajo es un aspecto de las condiciones de trabajo que impacta directamente en el desempeño de la labor de los agentes sanitarios 19,28,36,37. Dadas las características del ofrecimiento de la autotoma -para el cual los agentes sanitarios deben tomarse un tiempo para dialogar con la mujer, responder a dudas que puedan surgir y trasladar el tubo hasta el centro de salud- la estrategia tiene un importante potencial de sobrecarga de trabajo. El desarrollo de las actividades laborales de los agentes jujeños se enmarca dentro de la lógica del cumplimiento de metas, por lo que la incorporación de una nueva estrategia -en forma programática- incrementa la labor durante sus rondas; poniendo en tensión la relación entre la cantidad-calidad de los servicios que ofrecen a la comunidad. Con el objeto de cuidar a los agentes sanitarios como trabajadores de la salud, es relevante tener en cuenta este aspecto al momento de planificar sus tareas para que su desempeño y su vínculo con la comunidad o con el sistema de salud formal no resulten dañados.

Si bien este estudio aporta evidencia fundamental para la implementación de la estrategia de la autotoma en contexto programático, existen algunas limitaciones que deben ser consideradas. No todos los agentes sanitarios que participaron del Proyecto EMA contestaron la encuesta, quedando excluidos 64 agentes. Sin embargo, al comparar el grupo de agentes presentes con los ausentes, no se observaron diferencias significativas en cuanto a sexo y área. Por otro lado, se observó una mayor proporción de agentes del GAT, lo que podría resultar en un sesgo de selección. Ello implica que el grado de acuerdo, así como las percepciones reportadas por los agentes, podría diferir de lo encontrado en este análisis.

\section{Conclusión}

Los agentes sanitarios jujeños han sido los primeros en incorporar ofrecimiento de la autotoma como tarea programática en Argentina. Por tal motivo, conocer cuál es su perspectiva sobre la adopción de la estrategia posibilita detectar sus fortalezas y debilidades y, de ese modo, mejorar los circuitos para su implementación y/o extensión a otros contextos. En ese sentido, este estudio encontró un alto grado de acuerdo para incorporar la estrategia de la autotoma por parte de los agentes sanitarios jujeños, basado en el gran impacto que esta tecnología brinda a la prevención del CCU y al acceso/cuidado de la salud de las mujeres. Sin embargo, esta investigación encontró también que los agentes sanitarios demandan el acompañamiento del sistema de salud formal mediante acciones concretas, vinculadas a la capacitación y organización de la red de cuidado. Estas acciones son claves y fundamentales para equilibrar la sobrecarga de trabajo y, por lo tanto, evitar la insatisfacción laboral, el desgaste y la desmotivación de los agentes. 


\section{Colaboradores}

M. Curotto y P. Barletta participaron del análisis e interpretación de los datos, redacción del artículo y aprobación final para ser publicado. M. Paolino contribuyó con el análisis de los datos, revisión crítica del artículo y aprobación final para ser publicado. S. Arrossi, investigadora principal del estudio, contribuyó con su concepción original, el análisis de datos, redacción del artículo y su aprobación final para ser publicado. Los autores son responsables de todos los aspectos del trabajo, garantizando la exactitud e integridad de cualquier parte de la obra.

\section{Agradecimientos}

Las autoras quieren agradecer especialmente a los 191 agesntes sanitarios que participaron en el Proyecto EMA y a Laura Thouyaret, Milca Cuberli e Irina Perl por su participación en el proyecto. Instituto Nacional del Cáncer (Argentina) por la financiación.

\section{Referencias}

1. Ferlay J, Soerjomataram I, Ervik M, Dikshit R, Eser S, Mathers C, et al. GLOBOCAN 2012 v1.0: cancer incidence and mortality worldwide. Lyon: International Agency for Research on Cancer; 2013. (IARC CancerBase, 11).

2. Arrossi S, Paolino M. Proyecto para el mejoramiento del Programa Nacional de Prevención de Cáncer de Cuello Uterino en Argentina. Informe final: diagnóstico de situación del Programa Nacional y Programas Provinciales. Buenos Aires: Organización Panamericana de la Salud; 2008.

3. Arrossi S, Ramos S, Paolino M, Sankaranarayanan R. Social inequality in Pap smear coverage: identifying the women who are underuser of cervical cancer screening in Argentina. Reprod Health Matters 2008; 16:50-8.

4. Zamberlin N, Thouyaret L, Arrossi S. Lo que piensan las mujeres: conocimientos y percepciones sobre el cáncer de cuello de útero y realización del Pap. Buenos Aires: Organización Panamericana de la Salud, Organización Mundial de la Salud; 2011.

5. Gök M, Heideman D, van Kemenade FJ, Berkhof J, Rozendaal L, Spruyt J, et al. HPV testing on self collected cervicovaginal lavage specimens as screening method for women who do not attend cervical screening: cohort study. BMJ 2010; 340:c1040.

6. Giorgi Rossi P, Marsili LM, Camilloni L, Lossa A, Lattanzi A, Sani C, et al. The effect of selfsampled HPV testing on participation to cervical cancer screening in Italy: a randomised controlled trial (ISRCTN96071600). Br J Cancer 2011; 104:248-54.

7. Léniz J, Barriga MI, Lagos M, Ibáñez C, Puschel K, Ferreccio C. HPV vaginal self-sampling among women non-adherent to Papanicolaou screening in Chile. Salud Pública Méx 2013; 55:162-9.
8. Flores Y, Bishai D, Lazcano E, Shah K, Lörincz A, Hernández M, et al. Improving cervical cancer screening in Mexico: results from the Morelos HPV Study. Salud Pública Méx 2003; 45:38898.

9. Szarewski A, Cadman L, Mesher D, Austin J, Ashdown-Barr L, Edwards R, et al. HPV selfsampling as an alternative strategy in non-attenders for cervical screening - a randomised controlled trial. Br J Cancer 2011; 104:915-20.

10. Virtanen A, Nieminen P, Luostarinen T, Anttila A. Self-sample HPV tests as an intervention for nonattendees of cervical cancer screening in Finland: a randomized trial. Cancer Epidemiol Biomarkers Prev 2011; 20:1960-9.

11. Levy FM, Matos PE, Tomita NE. Programa de agentes comunitários de saúde: a percepção de usuários e trabalhadores da saúde. Cad Saúde Pública 2004; 20:197-203.

12. Galavote HS, Prado TN, Maciel EL, Lima RCD. Desvendando os processos de trabalho do agente comunitário de saúde nos cenários revelados na Estratégia Saúde da Família no município de Vitória (ES, Brasil). Ciênc Saúde Coletiva 2011; 16:231-40.

13. Silva JA, Dalmaso ASW. O agente comunitário de saúde e suas atribuições: os desafios para os processos de formação de recursos humanos em saúde. Interface Comun Saúde Educ 2002; 6:75-96.

14. Glenton C, Colvin CJ, Carlsen B, Swartz A, Lewin S, Noyes J, et al. Barriers and facilitators to the implementation of lay health worker programmes to improve access to maternal and child health: qualitative evidence synthesis. Cochrane Database Sys Rev 2013; (10):CD010414. 
15. Arrossi S, Thouyaret L, Herrero R, Campanera A, Magdaleno A, Cuberli M, et al. Effect of self-collection of HPV DNA offered by community health workers at home visits on uptake of screening for cervical cancer (the EMA study): a population-based cluster-randomised trial. Lancet Glob Health 2015; 3:e85-94.

16. Barbee L, Kobetz E, Menard J, Cook N, Blanco $\mathrm{J}$, Barton B, et al. Assessing the acceptability of self-sampling for HPV among haitian immigrant women: CBPR in action. Cancer Causes Control 2010; 21:421-31.

17. Mandingo M, Frett B, Laurent JR, Bishop I, Raymondville M, Marsh S, et al. Pairing community health workers with HPV self-sampling for cervical cancer prevention in rural Haiti. Int J Gynaecol Obstet 2015; 128:206:10.

18. Witmer A, Seifer SD, Finocchio L, Leslie J, O'Neil EH. Community health workers: integral members of the health care work force. Am J Public Health 1995; 85(8 Pt 1):1055-8.

19. Perry H. Final Report of Evidence Review Team 1. Which community support activities improve the performance of community health workers? A review of the evidence and of expert opinion with recommendations for policy, practice and research. Washington DC: Kaiser Family Foundation; 2012.

20. Andrade MAR. O trabalho do agente comunitário de saúde: relevância para o bom resultado da Estratégia da Saúde da Família. Lagoa Santa: Universidade Federal de Minas Gerais; 2012.

21. Arrossi S, Thouyaret L, Laudi R, Marín O, Ramírez J, Paolino M, et al. Implementation of HPV-testing for cervical cancer screening in programmatic contexts: the Jujuy demonstration project in Argentina. Int J Cancer 2015; 137:1709-18.

22. Drovetta RI. Prestadores de servicio de salud alopática y usuarios indígenas en la Puna de Atacama. In: Drovetta RI, Rodríguez ML, coordinadores. Padecimientos en grupos vulnerables del interior de Argentina: procesos históricos y actuales de salud, enfermedad y atención. Córdoba: Ferreyra Editor/Centro de Estudios Avanzados; 2010. p. 183-210.

23. Arbyn M, Verdoodt F, Snijders PJF, Verhoef VM, Suonio E, Dillner L, et al. Accuracy of human papillomavirus testing on self-collected versus clinician-collected samples: a metaanalysis. Lancet Oncol 2014; 15:172-83.

24. Arbyn M, Castle PE. Offering self-sampling kits for HPV testing to reach women who do not attend in the regular cervical cancer screening program. Cancer Epidemiol Biomarkers Prev 2015; 24:769-72.

25. Mbindyo PM, Blaauw D, Gilson L, English M. Developing a tool to measure health worker motivation in district hospitals in Kenya. Hum Resour Health 2009; 7:40.

26. Smith S, Deveridge A, Berman J, Negin J, Mwambene N, Chingaipe E, et al. Task-shifting and prioritization: a situational analysis examining the role and experiences of community health workers in Malawi. Hum Resour Health 2014; 12:24.
27. Bhattacharyya K, Winch P, LeBan K, Tien M Community health worker incentives and disincentives: how they affect motivation, retention and sustainability. Arlington: BASICS II; 2001.

28. Greenspan J, McMahon S, Chebet J, Mpunga M, Urassa D, Winch P. Sources of community health worker motivation: a qualitative study in Morogoro Region, Tanzania. Human Resour Health 2013; 11:52.

29. Giugliani C, Duncan BB, Harzheim E, Lavor AC, Lavor MC, Machado MM, et al. Community health workers programme in Luanda, Angola: an evaluation of the implementation process. Hum Resour Health 2014; 12:68.

30. Instituto Nacional del Cáncer. Consejería para la prevención del cáncer de cuello de útero. Propuesta para una mejor comunicación con las mujeres durante el tamizaje, seguimiento y tratamiento. Buenos Aires: Ministerio de Salud de la Nación; 2013.

31. Haq Z, Hafeez A. Knowledge and communication needs assessment of community health workers in a developing country: a qualitative study. Hum Resour Health. 2009; 7:59.

32. Kahssay HM, Taylor ME, Berman PA. Community health workers: the way forward. Geneva: World Health Organization; 1998. (Public Health in Action, 4).

33. Lehmann U, Sanders D. Community health workers: what do we know about them? The state of the evidence on programmes, activities, costs and impact on health outcomes of using community health workers. Geneva: World Health Organization; 2007.

34. Jaskiewicz W, Tulenko K. Increasing community health worker productivity and effectiveness: a review of the influence of the work environment. Hum Resour Health 2012; 10:38.

35. Zulu JM, Kinsman J, Michelo C, Hurtig AK. Integrating national community-based health worker programmes into health systems: a systematic review identifying lessons learned from low-and middle-income countries. BMC Public Health 2014; 14:987.

36. Javanparast S, Baum F, Labonte R, Sanders S. Community health workers' perspectives on their contribution to rural health and well-being in Iran. Am J Public Health 2011; 101:2287-92.

37. Li L, Hu H, Zhou H, He C, Fan L, Liu X, et al. Work stress, work motivation and their effects on job satisfaction in community health workers: a cross-sectional survey in China. BM Open 2014; 4:e004897. 


\section{Abstract}

The objective of this study was to analyze health agents' perception of self-collecting of samples for HPV testing among women and the degree of agreement by the agents to incorporate this approach into their daily tasks. A self-administered questionnaire was applied to 127/191 health agents that participated in the EMA Project (Proyecto Evaluación Modalidad Autotoma) in the province of Jujuy, Argentina, in 2012-2013. The health agents with and without the experience of offering self-collected sampling expressed a high degree of agreement towards adoption of the strategy (78.7\%), given its potential to prevent cervical cancer and its contribution to health care for the women under their coverage. However, the health agents identified the extra work and problems linking to the formal health system as the main barriers to offering this modality in the future. The study found that self-collecting of samples is a practice that can be adopted by health agents in the province of Jujuy, but that it should be accompanied by support measures from the formal health system.

Community Health Workers; Papillomaviridae; Uterine Cervical Neoplasms

\section{Resumo}

Este estudo objetivou analisar a percepção que possuem os agentes sanitários sobre o oferecimento da autoadministração do teste de HPV a mulheres e o nivel de compromisso dos agentes em incorporá-la a suas tarefas diárias. Para isso, foi aplicada uma pesquisa autoadministrada entre 127/191 agentes sanitários que participaram do Projeto EMA (Proyecto Evaluación Modalidad Autotoma), realizada na província de Jujuy (Argentina) no periodo 2012-2013. Os agentes sanitários que tiveram e não a experiência de oferecer a autoadministração manifestaram um alto grau de compromisso para a aprovação da estratégia (78,7\%), devido ao seu potencial para prevenir o câncer do colo do útero e os aportes que presenta para o cuidado da saúde das mulheres sob sua cobertura. No entanto, mostraram uma sobrecarga de trabalho e problemas de articulação com o sistema público de saúde, como os principais obstáculos para oferecer esta modalidade em um futuro. Este estudo encontrou que a autoadministração é una prática que pode ser adotada pelos agentes sanitários da província de Jujuy, no entanto deve ir acompanhada de ações de apoio por parte do sistema público de saúde.

Agentes Comunitários de Saúde; Papillomaviridae; Neoplasias do Colo do Útero
Recibido el 25/Ago/2015

Versión final presentada el 29/Mar/2016

Aprobado el 13/Jun/2016 